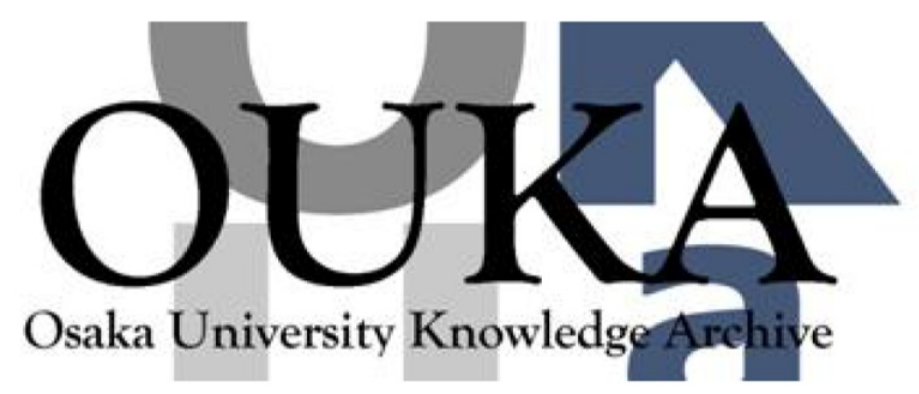

\begin{tabular}{|c|l|}
\hline Title & $\begin{array}{l}\text { Optical properties of } \\
\text { poly(disi lanyleneoligothienylene)s and their } \\
\text { doping characteristics }\end{array}$ \\
\hline Author(s) & Yoshino, K; Fujii, A.; Nakayama, H. et al. \\
\hline Citation & Journal of Applied Physics. 85(1) p. 414-p. 418 \\
\hline Issue Date & $1998-12-16$ \\
\hline oaire:version & VoR \\
\hline URL & https://hdl. handle. net/11094/75671 \\
\hline rights & \\
\hline Note & \\
\hline
\end{tabular}

Osaka University Knowledge Archive : OUKA

https://ir. Library. osaka-u. ac. jp/

Osaka University 


\section{Optical properties of poly(disilanyleneoligothienylene)s and their doping characteristics}

Cite as: Journal of Applied Physics 85, 414 (1999); https://doi.org/10.1063/1.369400

Submitted: 17 August 1998 . Accepted: 23 September 1998 . Published Online: 16 December 1998

K. Yoshino, A. Fujii, H. Nakayama, S. Lee, A. Naka, and M. Ishikawa
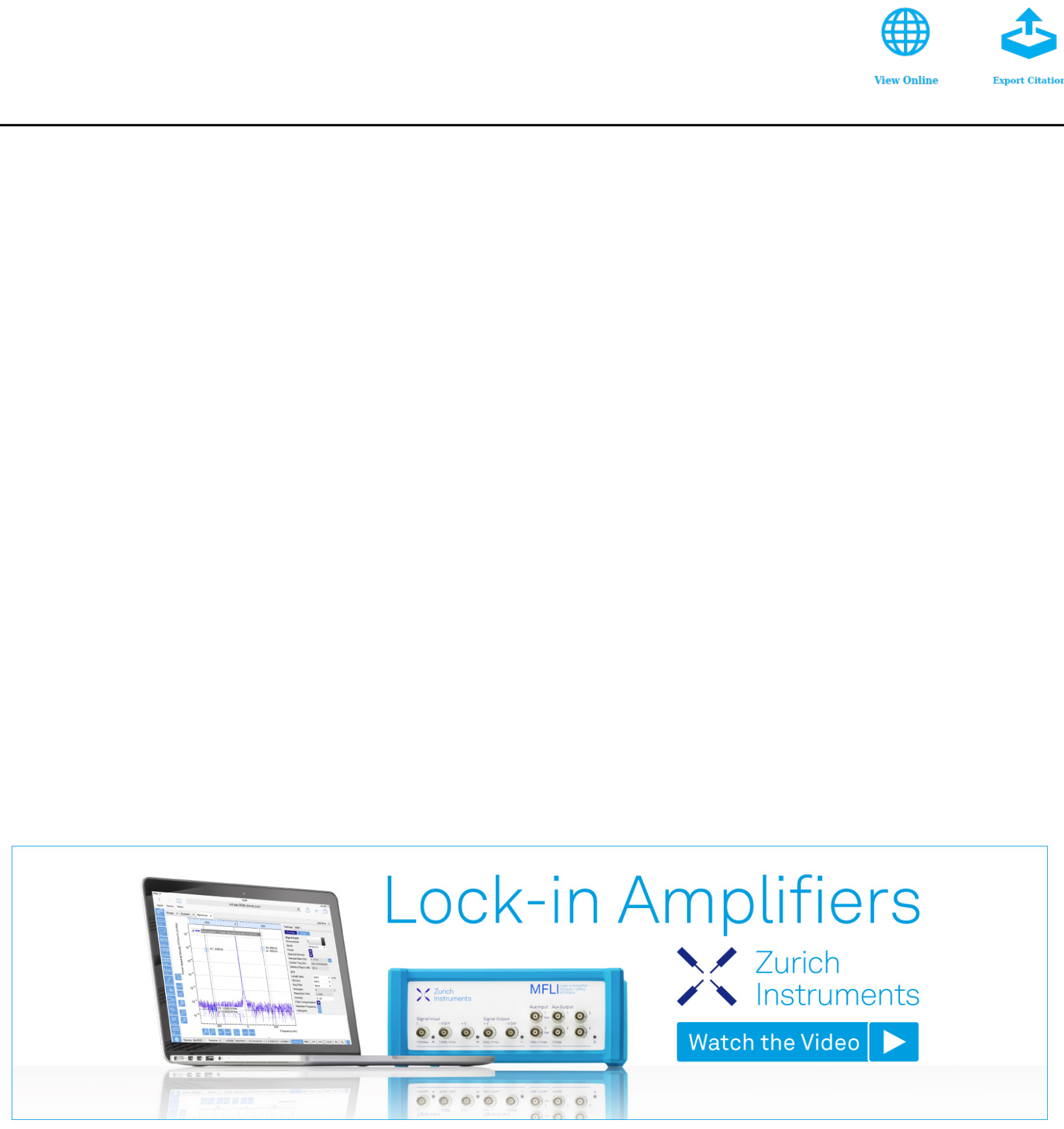


\title{
Optical properties of poly(disilanyleneoligothienylene)s and their doping characteristics
}

\author{
K. Yoshino, ${ }^{\text {a) }}$ A. Fujii, H. Nakayama, and S. Lee ${ }^{\text {b) }}$ \\ Department of Electronic Engineering, Faculty of Engineering, Osaka University, 2-1 Yamada-oka, Suita, \\ Osaka 565-0871, Japan
}

\begin{abstract}
A. Naka and M. Ishikawa
Department of Chemical Technology, Kurashiki University of Science and the Arts, 2640 Nishinoura, Tsurajima-cho, Kurashiki, Okayama 712-8001, Japan
\end{abstract}

(Received 17 August 1998; accepted for publication 23, September 1998)

\begin{abstract}
Optical absorption spectra, photoluminescence (PL), Stokes shift, and electroluminescence (EL) in poly(disilanyleneoligothienylene)s have been found to be dependent on the length of oligothienylene units. The color of EL changes from green to red with increasing length of oligothienylene from three to seven thiophene rings. Their stability is much higher than polysilanylene and increases with increasing length of oligothienylene and by $C_{60}$ doping. Photoconductivity is strongly enhanced and PL is remarkably quenched upon $C_{60}$ doping, which were discussed by the photo-induced electron transfer between aromatic units in these polymers and $C_{60}$. () 1999 American Institute of Physics. [S0021-8979(99)01501-7]
\end{abstract}

\section{INTRODUCTION}

Conducting polymers with highly extended $\pi$-electron systems in their main chains have attracted much interest from both fundamental and practical viewpoints, because of their novel physical phenomena and various new functional applications, such as insulator-metal transition upon doping, batteries ${ }^{1,2}$ electrochromism, ${ }^{3}$ electroluminescent (EL) devices. ${ }^{4,5}$ Recently, we reported on quenching of photoluminescence (PL) and enhancement of photoconductivity (PC) of conducting polymer upon $C_{60}$ doping. ${ }^{6}$

On the other hand, polysilanes, which are considered to be one-dimensional systems due to the delocalization of $\sigma$ orbitals along the Si backbone, have also attracted much attention, because of their various interesting phenomena such as doping effects, ${ }^{7}$ thermochromism, ${ }^{8}$ photoconductive response with large hole mobility and large nonlinear optical effects. We have also reported that PL was quenched upon $C_{60}$ doping in polysilanes with aromatic moieties in the side chain. ${ }^{9}$ Detection of electroluminescence in polysilanes was also reported on. ${ }^{10,11}$

Therefore, polymers containing silanylene and $\pi$-conjugated electron systems in their main chain are interesting from both scientific and practical points of view. Recently, we reported the unique properties of poly(disilanyleneoligophenylene)s (PDSiOP), the backbone main chain structures of which consist of disilanylene and oligophenylene units. Optical, electrical, electrochemical, $C_{60}$ doping and lasing properties of PDSiOP have been discussed. ${ }^{12-14}$

In this article, we report the novel optical and electrical properties such as photoluminescence and electrolumines-

\footnotetext{
a) Also at Institute for Fundamental Chemistry, 34-4 Takano-nishihiraki-cho, Sakyo-ku, Kyoto 606-8103, Japan; electronic mail: yoshino @ele.eng.osaka-u.ac.jp

${ }^{b)}$ On leave from Department of Thermophysics, Uzbek Academy of Sciences, Katartal 28, Chilanzer C, Tashkent 700135, Uzbekistan.
}

cence in poly(disilanyleneoligothienylene)s (PDSiOT) depending strongly on the length of oligothienylenes.

\section{EXPERIMENT}

PDSiOT, the molecular structures of which are shown in Fig. 1, were synthesized by the method already reported. ${ }^{15}$ That is, for example, PDSiOT with pentathienylene, poly(disilanylenepentathienylene), was prepared by crosscondensation of the di-Grignard reagent produced from 1,2-bis(5'-bromo-2,2' -bithien-5-yl)tetraethyldisilane with 2,5-dibromothiophene in the presence of the $\mathrm{Ni}$ (II) catalyst. These polymers thus obtained are soluble in common organic solvents such as chloroform and tetrahydrofuran, but the solubility decreased markedly with increasing the number of thienylene rings per disilanylene unit.

Thin films of these polymers were formed on quartz plates and ITO (In-Sn-oxide)-coated glass plates with a sheet resistance of $10 \Omega / \square$ by a spin coating method for measurements. Absorption and PL were measured utilizing spectrophotometer Hitachi 330 and spectrophotometer Hitachi F-2000, respectively. EL devices with the structures of $\mathrm{MgIn} / \mathrm{PDSiOT} / \mathrm{ITO}$ were fabricated by vacuum deposition of MgIn onto thin polymers films on ITO. EL measurements were also carried out by the method already reported. ${ }^{10-12}$ Photoconductivity and their doping effects were also studied

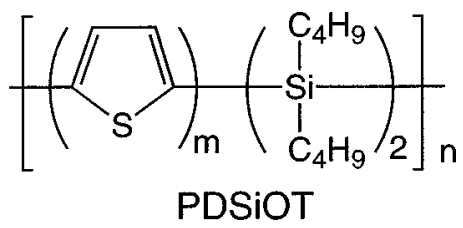

FIG. 1. Molecular structures of PDSiOT 


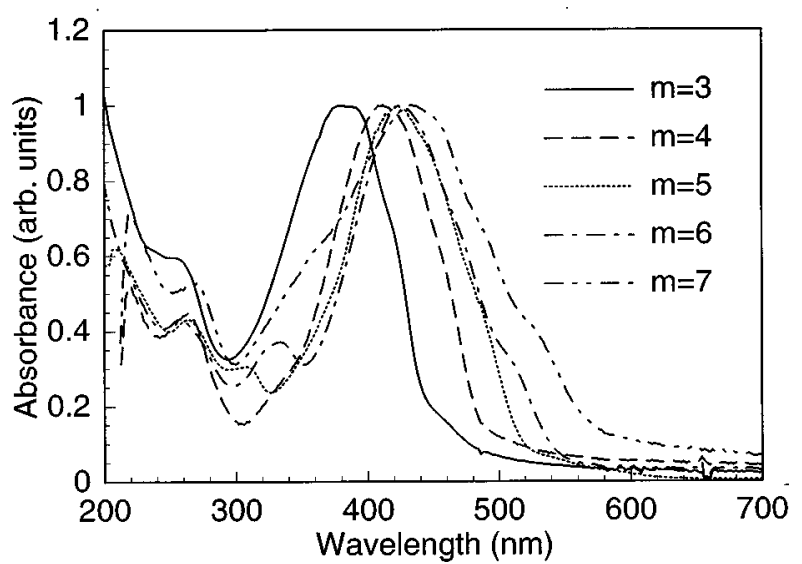

FIG. 2. Absorption spectra of PDSiOT as a function of oligothienylene length.

by the method already reported. ${ }^{9,13,14}$ Steady-state PC was measured by irradiation of the sample with Xe are lamp light passed through a monochromator.

Electrochemical measurement such as cyclic voltammetry and in situ spectral change upon electrochemical doping were carried out with three electrodes system containing working electrode with sample, a platinum counter electrode and a silver reference electrode in the electrolyte solution of tetrabutylammonium tetrafluoroborate $\left(\mathrm{TBABF}_{4}\right) /$ propylenecarbonate (PC) utilizing the Hokuto Denko HB105 function generator and the HA-105 potentiostat.

\section{RESULTS AND DISCUSSION}

Figure 2 shows the absorption spectra of PDSiOT with various lengths of thienylene rings. As evident from these figures, the absorption edge shifts to longer wavelength with increasing the number of thienylene rings in the main chain. From the analysis of the absorption spectral edge corresponding to highest occupied molecular orbital (HOMO)lowest unoccupied molecular orbital (LUMO) gap of PDSiOT, using the relationship of $(h \nu \times \alpha)^{2}$ vs $h \nu(\alpha$ : absorption coefficient) under the assumption of direct transition, the band-gap energies of PDSiOT were evaluated as $2.2-2.8 \mathrm{eV}$ depending on the number of thienylene length.

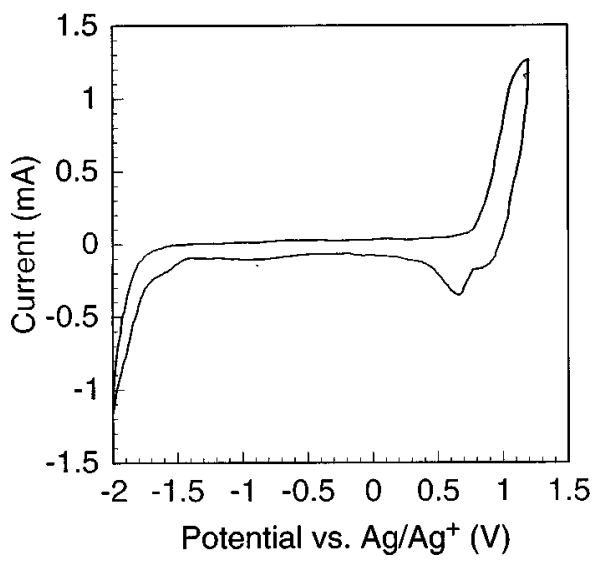

FIG. 3. Typical cyclic voltamogram of PDSiOT $(m=7)$.

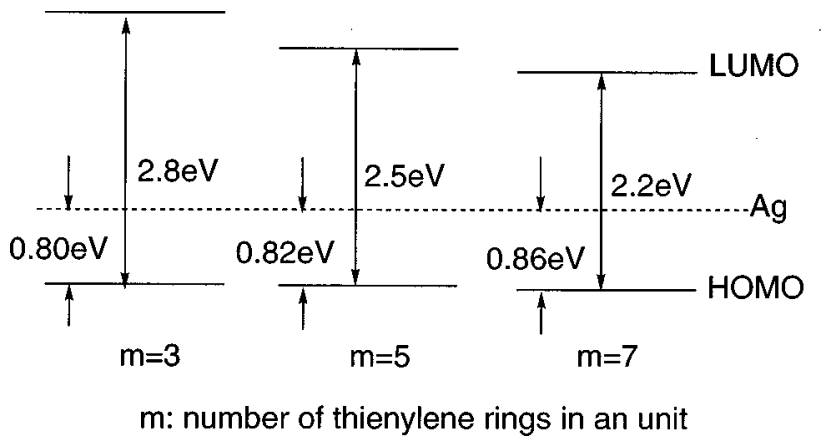

FIG. 4. Energy band diagram of PDSiOT.

Figure 3 shows the typical cyclic voltamogram of PDSiOT $(m=7)$ in $\mathrm{TBABF}_{4} / \mathrm{PC}$. The top of valence band (HOMO) was determined from the observed threshold potential of the electrochemical oxidation in the cyclic voltamogram. The electronic energy schemes in these polymers evaluated utilizing the absorption edges and the threshold in cyclic voltamogram are indicated in Fig. 4. As evident in this figure, the bottom of conduction band (LUMO) of PDSiOT becomes lower in energy with increasing length of thienylenes. On the other hand, lowering of HOMO with the length of thienylene is much less.

Corresponding to these red shifts of the band gap, the PL peaks also exhibit redshifts with increasing the number of thienylene rings as shown in Fig. 5. Figure 6 shows the dependence of the band gap and the energy of the PL peak on the number of thienylene rings. It should be mentioned that the PL intensity decreases with increasing the number of thienylene rings. The Stokes shift of PL peak from the absorption edge was also dependent on the number of thienylene rings, as shown in Fig. 7. That is, the Stokes shift decreases with increasing the number of thienylene rings. The Stokes shift in polythiophene film ${ }^{16}$ is small in comparison with PDSiOT as also evident in Fig. 7. These results suggest that the optical properties of PDSiOT in the visible range are determined by the oligothienylene moieties in the main chain.

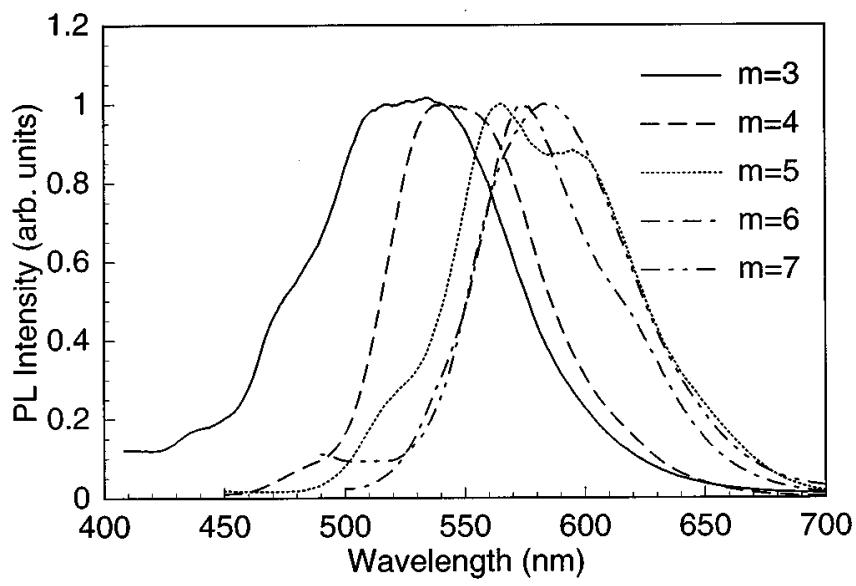

FIG. 5. Photoluminescence spectra of PDSiOT as a function of oligothienylene length. 


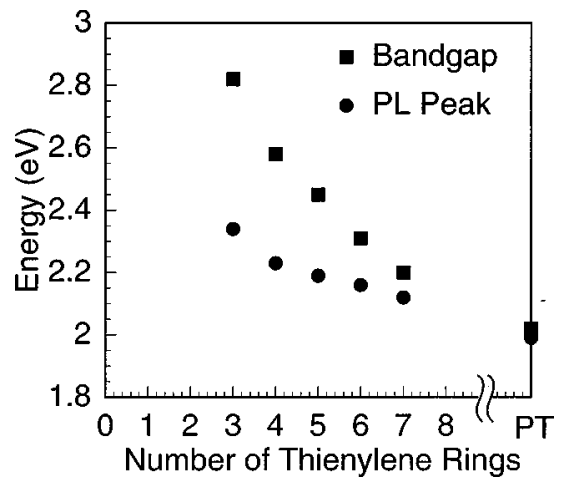

FIG. 6. Dependence of the bandgap and the energy of the PL peak on the number of thienylene rings. PT in this figure indicates polythiophene which includes large number of thienylene rings.

The film of oligothiophene formed by spin coating is not good enough quality for optical study. Therefore, the optical property of oligothiophene in solution was compared with those of PDSiOT in solution. Figure 8 shows the absorption and PL spectra of both oligothiophene and PDSiOT containing the same oligothiophene in its structure. It should be noted that the absorption edge and the wavelength of PL peak are different from those of PDSiOT with the same oligothiophene in its structure. It should also be mentioned that the Stokes shift in PDSiOT was larger than that of the same oligothiophene. This suggests that the $\mathrm{Si}$ atoms sandwiching oligothienylene influences on the electronic states of oligothienylenes and the relaxation of the main chain upon excitation.

Figure 9 indicates the absorption spectral change in PDSiOT $(m=6)$ upon electrochemical doping. As evident in this figure, upon doping, absorption peaks evolve in the spectral range longer in wavelength than the absorption edge, that is, in the energy range of the forbidden gap, which can be interpreted by the formation of polaronic state as the case of most conjugated polymers such as polythiophene. These spectral change upon doping supports the interpretation that the optical property of these polymers are determined by the oligothienylene units in the main chain.

It should also be mentioned that strong EL was also demonstrated utilizing these polymers as emitting layer in the EL device with a structure of MgIn PDSiOT/ITO. As

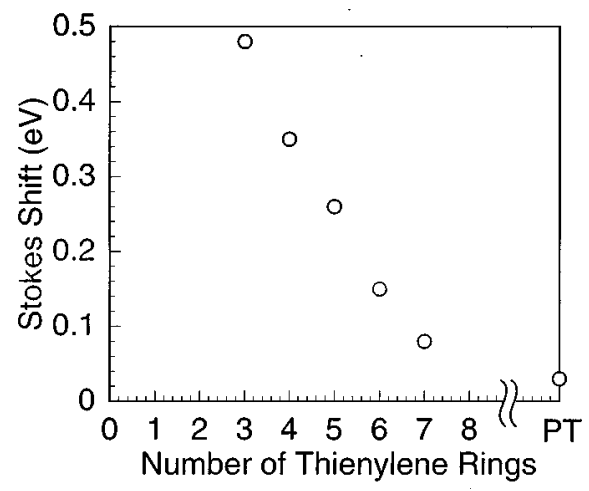

FIG. 7. Dependence of Stokes shift of PL peak from the absorption edge on the number of thienylene rings. PT in this figure indicates polythiophene.

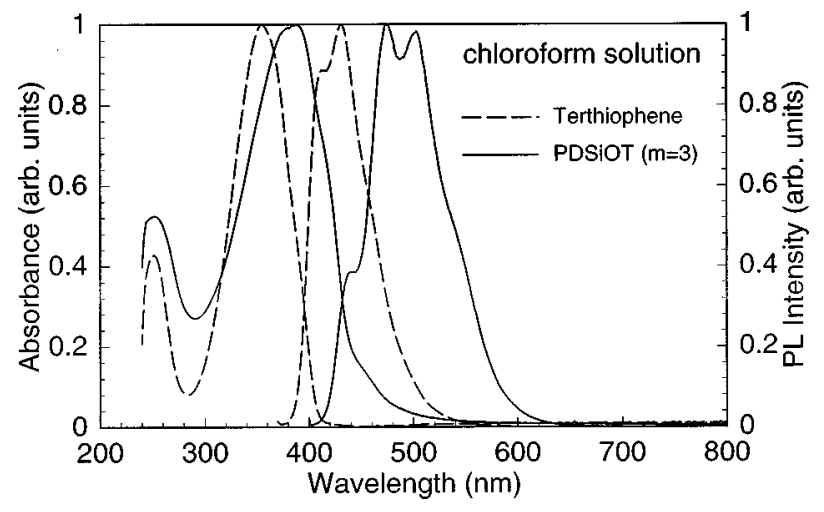

FIG. 8. Absorption and PL spectra of both oligothiophene and PDSiOT containing the same oligothiophene in its structure.

evident in Fig. 10, green EL was realized with PDSiOT ( $m$ $=3$ ) with terthienylene and the emission peak shifted with increasing the number of thienylene rings. PDSiOT with sixthienylene exhibited EL of red in color as also evident in Fig. 10. These facts also clearly indicate that the optical properties are mostly determined by the length of oligothienylenes.

The stability of these polymers and their emission characteristics are much higher than that of simple polysilanylenes. It should also be mentioned that the stability of these polymers increases with increasing the length of oligothienylene. This may be explained by stabilization of short $\mathrm{Si}-\mathrm{Si}$ bonds with side alkyl chains and by sandwiching between aromatic molecular units. The absorption of short wavelength light by aromatic units such as oligothienylenes may also result in the increase of stability.

The absorption spectrum of PDSiOT did not change markedly upon $C_{60}$ doping, which suggests that $C_{60}$ is not an effective dopant for PDSiOT in the ground state. However, the stability was still enhanced upon introduction of $C_{60}$ in these polymers. The increased stability to light irradiation upon $C_{60}$ doping suggests that the photoexcited species were killed by $C_{60}$, resulting in the suppression of photoinduced degradation.

The effects of $C_{60}$ doping in PDSiOT under dark conditions are small. However, optical properties are influenced

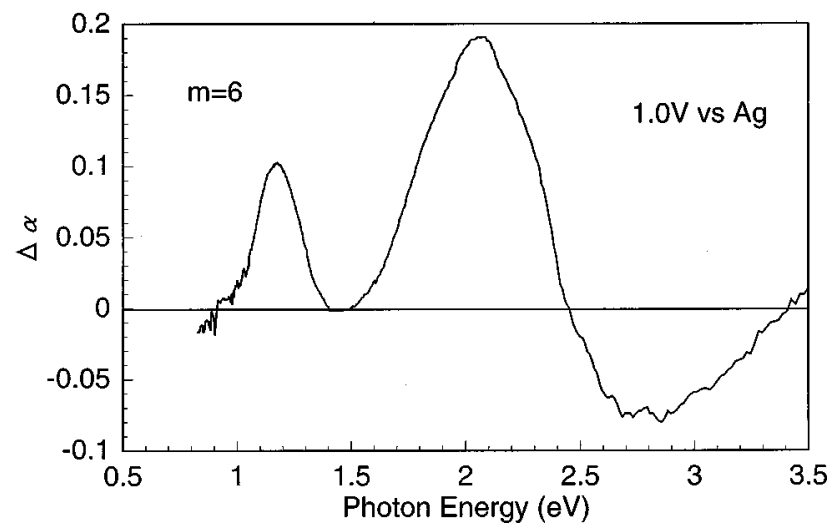

FIG. 9. Change of the absorption spectrum of PDSiOT $(m=6)$ taken in situ during electrochemical $p$-type doping. 


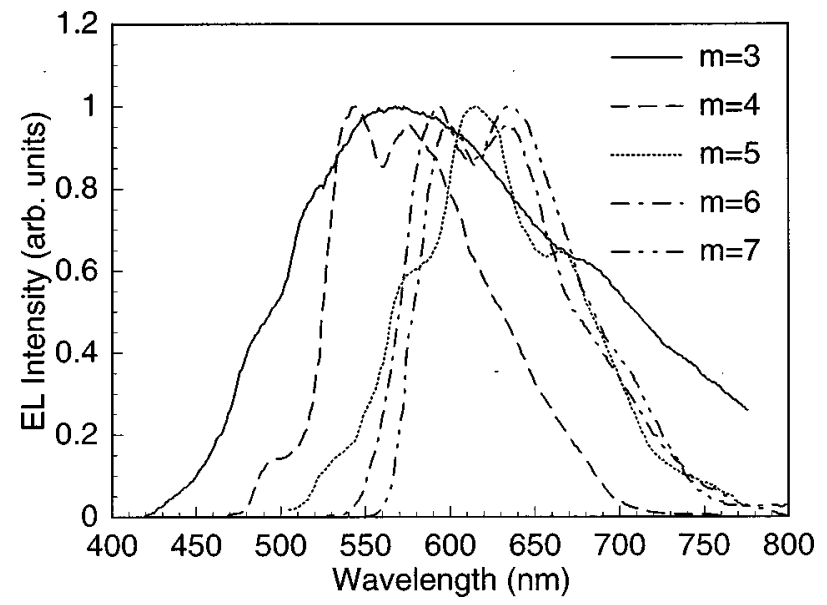

FIG. 10. Electroluminescence spectra of PDSiOT as a function of oligothienylene length.

strongly by $C_{60}$ doping. For example, the PL intensity of PDSiOT $(m=5)$ excited by violet light at $450 \mathrm{~nm}$ changed markedly upon $C_{60}$ doping as shown in Fig. 11. Though the wavelength of the emission peak did not change markedly, its intensity decreased drastically with increasing $C_{60}$ concentration.

As shown in Fig. 12, PC of PDSiOT $(m=5)$ was enhanced significantly upon $C_{60}$ doping. The photoconduction response at the photon energy corresponding to the band-gap energy of PDSiOT was notably enhanced by the introduction of a small amount of $C_{60}$. This observation of enhancement of PC in PDSiOT upon $C_{60}$ doping can also be explained by photoinduced charge transfer from the excitons in PDSiOT to $C_{60}$ by formation of stable $C_{60}^{-1}$. The remaining positive carriers on the main chain of PDSiOT contribute to enhanced photoconduction. $^{14}$

The HOMO of PDSiOT is lower than the LUMO of $C_{60}$, as shown in Fig. 13. Therefore, electron transfer from PDSiOT to $C_{60}$ is difficult in the ground state which is consistent with the small changes in the absorption spectrum in PDSiOT by $C_{60}$ doping under dark conditions.

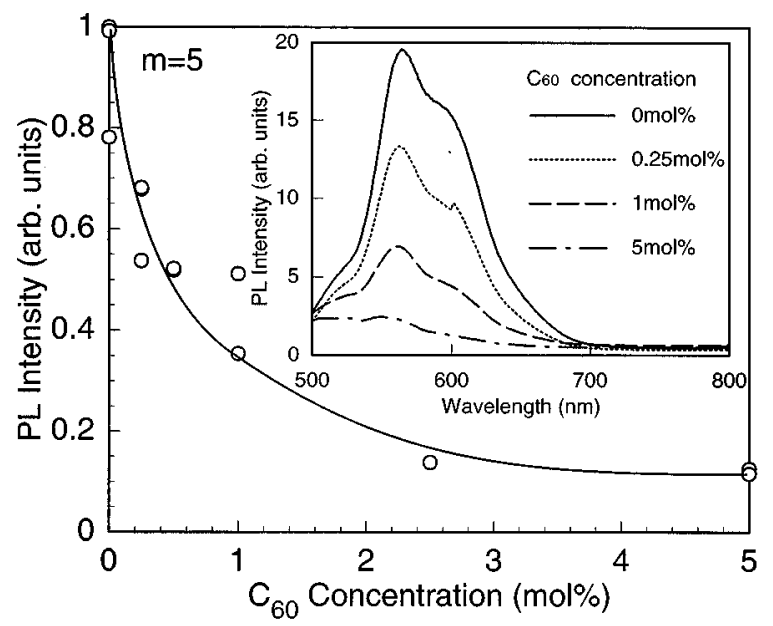

FIG. 11. Dependence of photoluminescence intensity of PDSiOT $(m=5)$ on the concentration of $C_{60}$. Inset shows photoluminescence spectra of PDSiOT $(m=5)$ with $C_{60}$ doping.

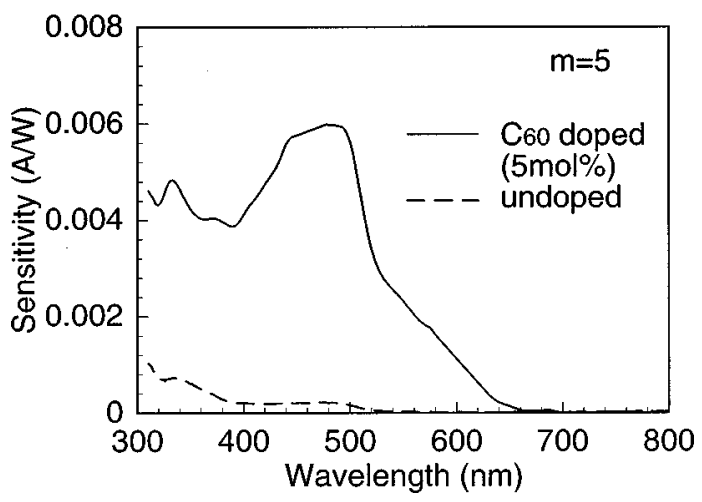

FIG. 12. Photoconduction spectra of PDSiOT $(m=5)$ as a function of $C_{60}$ concentration.

Upon irradiation of light with a photon energy exceeding the band-gap energy of PDSiOT $(m=5), 2.5 \mathrm{eV}$, the interband optical transition will create excitons in PDSiOT. When excitons migrating along the main chain of PDSiOT encounter with $C_{60}$, electrons are transferred to $C_{60}$, resulting in dissociation of excitons and hence quenching of PL and also enhancement of PC due to migration of remaining positive carriers on the polymer chain as schematically shown in Fig. 13.

We have already reported that in polysilanes with saturated hydrocarbon side chains, PL quenching was not clearly
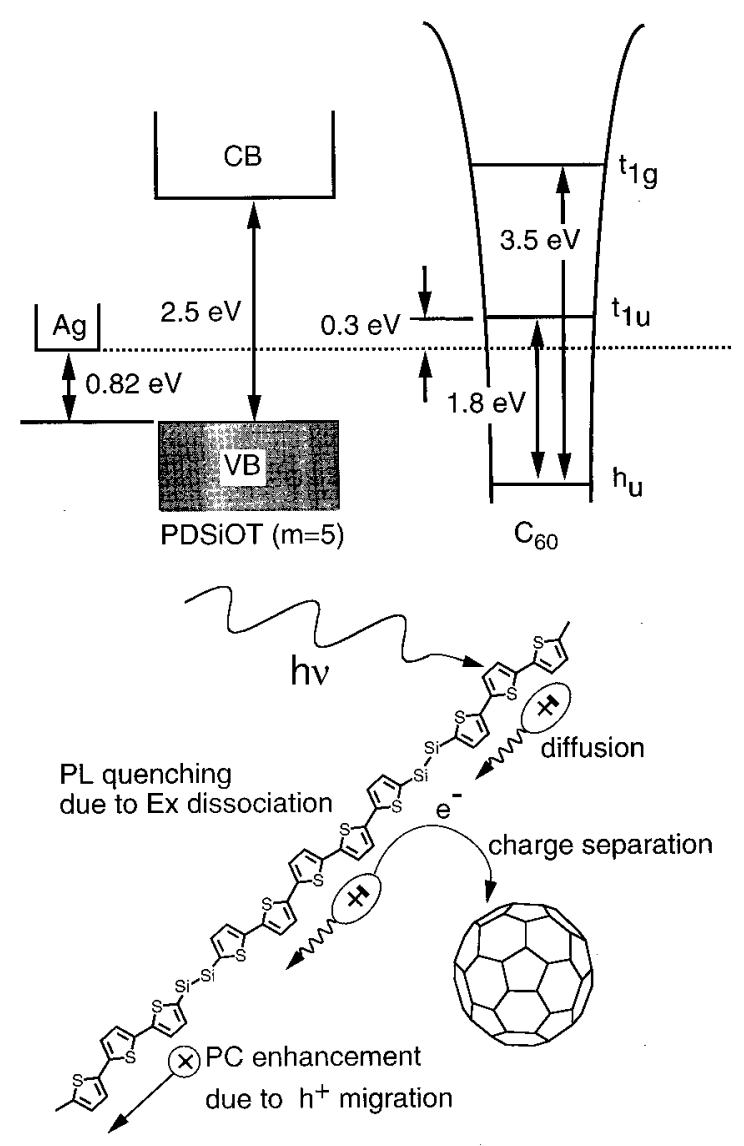

FIG. 13. Schematical explanation of photoinduced charge transfer between PDSiOT and $C_{60}$. 
observed but clear PL quenching was confirmed to occur in polysilanes with aromatic units in the side chain. ${ }^{9}$ In that case, aromatic units are interpreted to play a role as mediators of charge transfer, which is consistent with the present observation of the important role of thienylene units in PDSiOT for $C_{60}$ doping.

\section{SUMMARY}

Absorption and PL spectra in PDSiOT exhibit remarkable redshift with increasing the number of thienylene rings. The Stokes shift also depends on the number of thienylene rings. The color of EL changes from green to red with increasing number of thienylene rings from three to seven. The stability was still enhanced upon $C_{60}$ doping. PL was remarkably quenched and photoconductivity was strongly enhanced upon $C_{60}$ doping, which were explained by the photoinduced electron transfer between aromatic units in these polymers and $C_{60}$. These facts also indicate that the properties of these polymers are mainly determined by the aromatic units such as oligothienylene in the main chain.

\section{ACKNOWLEDGMENT}

Part of the work was financially supported by the Research for the Future Program of the Japan Society for the Promotion of Science (Project No. JSPS-RFTF96P00206).
${ }^{1}$ P. J. Nigrey, D. MacInnes, Jr., D. P. Nairns, A. G. MacDiarmid, and A. J. Heeger, J. Electrochem. Soc. 128, 1651 (1981).

${ }^{2}$ K. Kaneto, K. Yoshino, and Y. Inuishi, Jpn. J. Appl. Phys., Part 2 22, L157 (1983)

${ }^{3}$ K. Yoshino, K. Kaneto, and Y. Inuishi, Jpn. J. Appl. Phys., Part 2 22, L567 (1983)

${ }^{4}$ J. H. Burroughes, D. D. C. Bradley, A. R. Brown, R. N. Marks, K. Mackay, R. H. Friend, P. L. Burns, and A. B. Holmes, Nature (London) 347, 539 (1990).

${ }^{5}$ Y. Ohmori, M. Uchida, K. Muro, and K. Yoshino, Jpn. J. Appl. Phys., Part 2, 30, L1941 (1991).

${ }^{6}$ S. Morita, A. A. Zakhidov, and K. Yoshino, Solid State Commun. 82, 249 (1992).

${ }^{7}$ R. West, L. D. David, P. I. Djurovich, K. L. Stearly, K. S. V. Srinivasan, and H. Yu, J. Am. Chem. Soc. 103, 7352 (1981).

${ }^{8}$ K. Yokoyama and M. Yokoyama, Solid State Commun. 70, 241 (1989).

${ }^{9}$ K. Yoshino, K. Yoshimoto, M. Hamaguchi, T. Kawai, A. A. Zakhidov, H. Ueno, M. Kakimoto, and H. Kojima, Jpn. J. Appl. Phys., Part 2 34, L141 (1995).

${ }^{10}$ A. Fujii, K. Yoshimoto, M. Yoshida, Y. Ohmori, and K. Yoshino, Jpn. J. Appl. Phys., Part 2 34, L1365 (1995).

${ }^{11}$ A. Fujii, K. Yoshimoto, M. Yoshida, Y. Ohmori, K. Yoshino, H. Ueno, M. Kakimoto, and H. Kojima, Jpn. J. Appl. Phys., Part 1 35, 3914 (1996).

${ }^{12}$ K. Yoshino, K. Hosoda, A. Fujii, and M. Ishikawa, Jpn. J. Appl. Phys., Part 2 36, L368 (1997).

${ }^{13}$ K. Hosoda, K. Tada, M. Ishikawa, and K. Yoshino, Jpn. J. Appl. Phys., Part 2 36, L372 (1997).

${ }^{14}$ K. Yoshino, K. Tada, M. Hirohata, R. Hidayat, S. Tatsuhara, M. Ozaki, A. Naka, and M. Ishikawa, Jpn. J. Appl. Phys., Part 2 36, L1548 (1997).

${ }^{15}$ J. Ohshita, T. Watanabe, D. Kanaya, H. Ohsaki, M. Ishikawa, H. Ago, K. Tanaka, and T. Yamabe, Organometallics 13, 5002 (1994).

${ }^{16}$ K. Kaneto, Y. Kohno, and K. Yoshino, Solid State Commun. 51, 267 (1984). 